\title{
Iliolumbar Vein
}

National Cancer Institute

\section{Source}

National Cancer Institute. Iliolumbar Vein. NCI Thesaurus. Code C53048.

A blood vessel running parallel to the iliolumbar artery connected to the deep circumflex iliac and lumbar veins that drains into the internal iliac vein. 